\title{
Reduction of Perioperative Antibiotic Prophylaxis in Open Radical Cystectomy with Ileal Conduit Is Feasible: Results of a Prospective Clinical Trial
}

\author{
Laila Schneidewind Laura Torabi Desiree L. Dräger Oliver W. Hakenberg \\ Department of Urology, University Medical Center Rostock, Rostock, Germany
}

\section{Mini-Summary}

- First prospective study of the colonization of ileal conduit (IC) following radical cystectomy (RC).

- Evaluation of reduction of antibiotic prophylaxis to single-shot antibiosis in RC with IC could be feasible.

- Patients undergoing palliative cystectomy with IC are at highest risk for developing infectious complications.

\section{Keywords}

Muscle-invasive bladder cancer $\cdot$ Radical cystectomy $\cdot$ lleal conduit · Antibiotic stewardship

\begin{abstract}
Introduction: The aim of this study is to perform a prospective clinical trial in antibiotic prophylaxis, infectious complication, and colonization of ileal conduit (IC) following radical cystectomy (RC) since urinary tract infections (UTIs) and surgical site infections (SSIs) contribute significantly to the morbidity associated with RC and IC. Moreover, an optimal regimen of antibiotic prophylaxis has not been established, yet. Material and Methods: After a positive vote of ethical review committee and the registration at the German Clinical Trials Register (DKRS 00020406), we started a prospective clinical unicentric not interventional study. The urine samples were collected by sterile catheterization of the IC. All patients received an antibiotic prophylaxis with $3 \times 500 \mathrm{mg}$ metronidazole and $3 \times 1.5 \mathrm{~g}$ cefuroxime intravenously for 3 days starting on the day before RC. Ureteral stents got re-
\end{abstract}

moved on days 9 and 10 after surgery without prior antibiotic administration. The student $t$ test and the $x^{2}$ test or the Fisher exact test were used. For risk factor assessment, the univariate Cox regression method was applied. Results: Nineteen male (63.3\%) and 11 female patients (36.7\%) with a median age of 70.5 years were included. Three patients developed complicated UTI (10\%) on day 12 after RC with $E$. faecium and needed antibiotic treatment with meropenem (Clavien-Dindo II). Two patients (6.7\%) developed SSI with E. faecium and needed surgery (Clavien-Dindo IIIb). Palliative RC $(p<0.0001)$, prior radiation therapy $(p<0.0001)$, and timeframe $>3$ months from diagnosis to $\mathrm{RC}(p=0.036)$ are significantly associated with the development of complicated UTI. Interestingly, the IC got colonized with Staph. haemolyticus at day 12 after RC ( $n=12 ; 40.0 \%)$. We must assume that our data have some limitations like a unicentric study population. Conclusion: Further evaluation of reduction to single-shot antibiotic prophylaxis in nonpalliative RC with IC could be feasible.

(c) 2021 The Author(s)

Published by S. Karger AG, Basel
C 2021 The Author(s)

Published by S. Karger AG, Basel

This is an Open Access article licensed under the Creative Commons Attribution-NonCommercial-4.0 International License (CC BY-NC) (http://www.karger.com/Services/OpenAccessLicense), applicable to the online version of the article only. Usage and distribution for commercial purposes requires written permission.
Correspondence to:

Laila Schneidewind, laila.schneidewind@med.uni-rostock.de 


\section{Introduction}

Radical cystectomy (RC) with urinary diversion is the established standard of care for treating high-risk and muscle-invasive bladder cancer [1-3]. Ileal conduit (IC) is the most commonly used urinary diversion since it is considered cost-effective, clinically satisfactory, and a reliable solution in terms of long-term outcome $[1,4]$. Urinary tract infections (UTIs), sepsis, and surgical site infection (SSI) contribute significantly to the morbidity associated with RC and IC during inpatient treatment or in the first 30 days following surgery. Furthermore, in a population-based study in the USA, the overall infectious event rate after RC was 25\% [5]. However, reduction of postoperative infections necessitates appropriate perioperative antimicrobial prophylaxis targeting causative bacteria, but an optimal antibiotic prophylaxis regimen has not been established, yet [6]. The main reasons that an optimal regimen of antibiotic prophylaxis has not been described are the antibiotic prophylaxis practices are highly heterogenous in RC, there is a lack of adherence to published guidelines, and knowledge about antibiotic prophylaxis in RC, especially with IC as diversion, mostly arises from retrospective case series [5, 7-11]. Indeed, due to rising antibiotic resistance rates worldwide, the knowledge about an optimal antibiotic prophylaxis regimen is more important than ever. Additionally, this is an essential step to antibiotic stewardship [12,13].

Consequently, we conducted a prospective unicentric clinical trial about antibiotic prophylaxis in RC with IC, infectious complications, and colonization of the IC during inpatient treatment. The primary endpoints of this study were infectious complications during inpatient treatment and the accurate description of colonization of the IC by sterile catheterization on defined timepoints. The secondary endpoints were the identification of associations of clinical parameters with infectious complications and the identification of risk factors for these complications.

\section{Material and Methods}

\section{Development of the Study and Study Population}

The study was designed according to the guidelines in the synthesis of qualitative research (ENTREQ) found on the equatornetwork.org [14]. Before starting the study, we obtained the approval of the local ethics review board at the University Medical Center in Rostock (A 2019-0213 from 26 November 2019). Furthermore, the study was registered at the German Clinical Trials Register (DKRS 00020406). Formally, this study is a prospective unicentric noninterventional trial. The inclusion criteria were adult patient (aged over 18 years) and RC with IC as urinary diversion due to muscle-invasive bladder cancer. If the inclusion criteria were met and informed consent was obtained from the patient, there were no further exclusion criteria. All relevant patient data like patient history, demographic characteristics, and all relevant clinical data were prospectively collected according to the study protocol, available via DKRS number, on defined timepoints (preoperatively and days 4,8 , and 12 postoperatively). The urine samples were collected by sterile catheterization of the IC, except prior to RC when midstream urine was used. All patients received an antibiotic prophylaxis with $3 \times 500 \mathrm{mg}$ metronidazole and $3 \times 1.5 \mathrm{~g}$ cefuroxime intravenously for 3 days starting on the day before RC. No bowel preparation was done. RC with bilateral pelvic lymphadenectomy was performed according to the established surgical principles and IC was implemented with a $12-15-\mathrm{cm}$ ileal loop taken $20 \mathrm{~cm}$ from the ileocecal valve, with end-to-end ileostomy and separate end-to-side anastomoses of the ureters to the IC. Ureteric stents got removed on days 9 and 10 after surgery without prior antibiotic administration. We applied the principles of enhanced recovery after surgery (fast-track surgery). The patients got discharged from the hospital on day 12 following RC, when no complication was obvious or intravenous treatment was necessary.

\section{Definitions and Statistical Analysis}

To classify postoperative complications, we used the ClavienDindo classification [15]. The 2017 tumor, node, and metastasis classification was used for staging. For grading, the 1973 and 2004/2016 WHO grading classification were utilized [16]. Furthermore, we considered $10^{5}$ colony forming units/mL to be significant. Chronic kidney failure was defined according to the Kidney Disease Improving Global Outcome clinical practice guidelines [17]. Definitions of UTI or complicated UTI were all used according to the European Association of Urology guideline [18]. Additionally, sepsis was defined as bacterial infection plus the criteria of systemic inflammatory response syndrome (SIRS). If the blood culture was negative, all 4 of the SIRS criteria needed to be fulfilled to diagnose sepsis. If the blood culture was positive, 2 of the SIRS criteria were enough to diagnose sepsis [19].

For each numeric variable, the numeric distribution was preliminarily assessed by the Kolmogorov-Smirnov test. Descriptive statistics were made with mean and standard deviation for normal distribution or with median and IQR for nonparametric data. For parametric continuous variables, the Student's $t$ test was used, and for parametric categorical variables, the $\chi^{2}$ test or the Fisher exact test was used. For risk factor assessment, the univariate Cox regression method was used, and the significance was tested with the Wald statistic. All reported $p$ values were based on a 2 -sided hypothesis; $p<0.05$ was considered to be significant. All statistical calculations were performed using a statistical package for the Social Sciences 26.0 software (SPSS Inc., Chicago, IL, USA).

\section{Results}

Demographic Characterization of the Study Population

We included 30 adult patients (from May 2020 until January 2021; 9 months study period) with a median age 
Table 1. Demographic characterization of the study population $(n=30)$

\begin{tabular}{|c|c|c|}
\hline Parameter & $N(\%)$ & Median (IQR) \\
\hline Age & - & $70.5(62.0-79.0)$ \\
\hline \multicolumn{3}{|l|}{ Sex } \\
\hline Male & $19(63.3)$ & \multirow{2}{*}{-} \\
\hline Female & $11(36.7)$ & \\
\hline Charlson Comorbidity Index & - & $2.0(1.0-3.5)$ \\
\hline \multicolumn{3}{|l|}{ Histology } \\
\hline Urothelial carcinoma & $23(76.7)$ & \multirow{5}{*}{-} \\
\hline Squamous cell carcinoma & $3(10.0)$ & \\
\hline Adenocarcinoma & $1(3.3)$ & \\
\hline Neuroendocrine carcinoma & $1(3.3)$ & \\
\hline Prostate cancer & $2(6.7)$ & \\
\hline \multicolumn{3}{|l|}{ Tumor status } \\
\hline pT1 & $4(13.3)$ & \multirow{7}{*}{-} \\
\hline pT2a & $4(13.3)$ & \\
\hline $\mathrm{pT} 2 \mathrm{~b}$ & $3(10.0)$ & \\
\hline pT3a & $2(6.7)$ & \\
\hline pT3b & $8(26.7)$ & \\
\hline pT4a & $7(23.3)$ & \\
\hline pT4b & $2(6.7)$ & \\
\hline \multicolumn{3}{|l|}{ Nodal status } \\
\hline NO & $18(60.0)$ & \multirow{3}{*}{-} \\
\hline N1 & $6(20.0)$ & \\
\hline N2 & $6(20.0)$ & \\
\hline \multicolumn{3}{|l|}{ Metastasis status } \\
\hline cM0 & $28(93.3)$ & \multirow{3}{*}{-} \\
\hline cM1 & $2(6.7)$ & \\
\hline & Both bone metastasis & \\
\hline \multicolumn{3}{|l|}{ Lympho-vascular-invasion status } \\
\hline LO & $17(56.7)$ & \multirow{2}{*}{-} \\
\hline L1 & $13(43.3)$ & \\
\hline \multicolumn{3}{|l|}{ Vascular-invasion status } \\
\hline V0 & $23(76.7)$ & \multirow{2}{*}{-} \\
\hline V1 & $7(23.3)$ & \\
\hline \multicolumn{3}{|l|}{ Perineural-invasion status } \\
\hline $\mathrm{Pn} 0$ & $20(66.7)$ & \multirow{2}{*}{ - } \\
\hline Pn1 & $10(33.3)$ & \\
\hline \multicolumn{3}{|l|}{ R status (surgical margins) } \\
\hline Ro & $20(66.7)$ & \multirow{2}{*}{-} \\
\hline $\mathrm{R} 1$ & $10(33.3)$ & \\
\hline \multicolumn{3}{|l|}{ Grading } \\
\hline G1 & $0(0)$ & \multirow{5}{*}{-} \\
\hline $\mathrm{G} 2$ & $7(23.3)$ & \\
\hline G3 & $22(73.3)$ & \\
\hline & Including 2 Gleason 9 & \\
\hline G4 & $1(3.3)$ & \\
\hline
\end{tabular}

of 70.5 years. Muscle-invasive urothelial carcinoma of the bladder was the most frequent underlying disease $(n=23 ; 76.7 \%)$. Table 1 gives an overview of the demographic characteristics of the study population. The median time from initial diagnosis to RC was 1.0 months (IQR 1.0-2.0 months). Two patients received (6.7\%) palliative $\mathrm{RC}$; both had bone metastasis prior to $\mathrm{RC}$ and already had received radiation therapy. Another 2 patients
(6.7\%) underwent neoadjuvant chemotherapy with gemcitabine/cisplatin prior to RC. Concerning the perioperative management, the median operation time was 210 min (IQR 182.5-238.0 min). The median transfusion rate of packed red blood cells was 0 (IQR $0-2$ ), and no fresh frozen plasma products or packed platelets were transfused. 

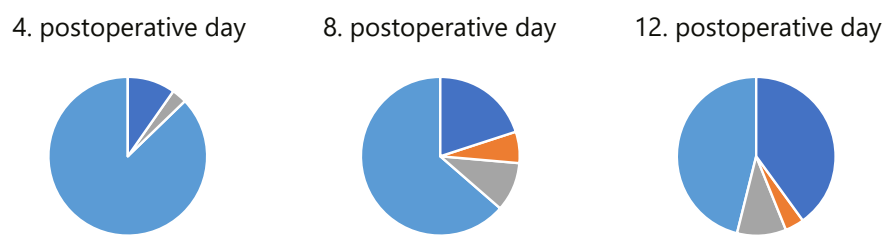

Staphylococcus haemolyticus

Staphylococcus epidermidis

- Candida albicans

Enterococcus faecium

Sterile culture

Fig. 1. Urine culture of IC with at least $10^{5} \mathrm{CFU}$ or no pathogen detection (sterile). IC, ileal conduit; CFU, colony forming units.

Table 2. Associations with complicated UTI and SSIs $(n=30)$

\begin{tabular}{|c|c|c|c|}
\hline Parameter & $N(\%)$ & $\begin{array}{l}\text { Association with } \\
\text { complicated UTI } \\
p \text { value }\end{array}$ & $\begin{array}{l}\text { Association } \\
\text { with SSI } \\
p \text { value }\end{array}$ \\
\hline Complicated UTI & $\begin{array}{l}3(10.0) \\
\text { All with E. faecium }\end{array}$ & - & - \\
\hline SSI & $\begin{array}{l}2(6.7) \\
\text { All with E. faecium }\end{array}$ & - & - \\
\hline Palliative cystectomy & $2(6.7)$ & $<0.001$ & 0.700 \\
\hline Age $>70$ years & $15(50.0)$ & 0.073 & 0.150 \\
\hline Charlson Comorbidity Index $>2$ & $10(33.3)$ & 0.204 & 0.309 \\
\hline Operation time $>200 \mathrm{~min}$ & $17(56.7)$ & 0.717 & 0.208 \\
\hline Perioperative transfusion & $13(43.3)$ & 0.398 & 0.100 \\
\hline Chronic kidney failure & $5(16.7)$ & 0.422 & 0.520 \\
\hline Diabetes mellitus type II & $7(23.3)$ & 0.066 & 0.520 \\
\hline Prior pelvic irradiation & $2(6.7)$ & $<0.001$ & 0.700 \\
\hline $\begin{array}{l}\text { Neoadjuvant chemotherapy with gemcitabine/ } \\
\text { cisplatin }\end{array}$ & $2(6.7)$ & 0.631 & 0.700 \\
\hline Time from diagnosis to $\mathrm{RC}>3$ months & $6(20.0)$ & 0.036 & 0.280 \\
\hline
\end{tabular}

UTI, urinary tract infection; SSI, surgical site infection; RC, radical cystectomy.

Infectious Complications, Associations with Patient

Data, and Identification of Risk Factors

Three patients developed complicated UTI (10\%) on day 12 following $\mathrm{RC}$ and after removing the ureteric stents with E. faecium, so they needed antibiotic treatment with meropenem (Clavien-Dindo II). Two patients (6.7\%) developed SSI with E. faecium, also on day 12 following RC, so they needed surgery (secondary wound closure and lavage, Clavien-Dindo IIIb). None of the 5 patients (16.7\%) with infectious complications fulfilled the defined sepsis criteria. The remaining 25 patients $(83.3 \%)$ were discharged from the hospital on day 12 following RC, and these patients had no second hospital admission in the first 30 days following RC again. Table 2 gives an overview of associations with patient data and complicated UTI as well as SSI. Palliative RC $(p<0.001)$, prior radiation therapy $(p<0.001)$, and timeframe $>3$ months from diagnosis to RC $(p=0.036)$ are significantly associated with the development of complicated UTI. No significant association with patient data and SSI was found. In univariate Cox regression, we could not identify a single risk factor for UTI, but we were able to identify 1 significant risk factor for SSI, namely, operation time in minutes (HR 1.74; 95\% CI 0.87-1.2; $p=0.001$ ).

\section{Colonization of the IC and Development of the}

Laboratory Parameters during Inpatient Treatment

Prior to RC, no patient had significant bacteriuria in midstream urine. The IC gets colonized with bacteria or fungi of the normal skin flora over time, for example, IC is 


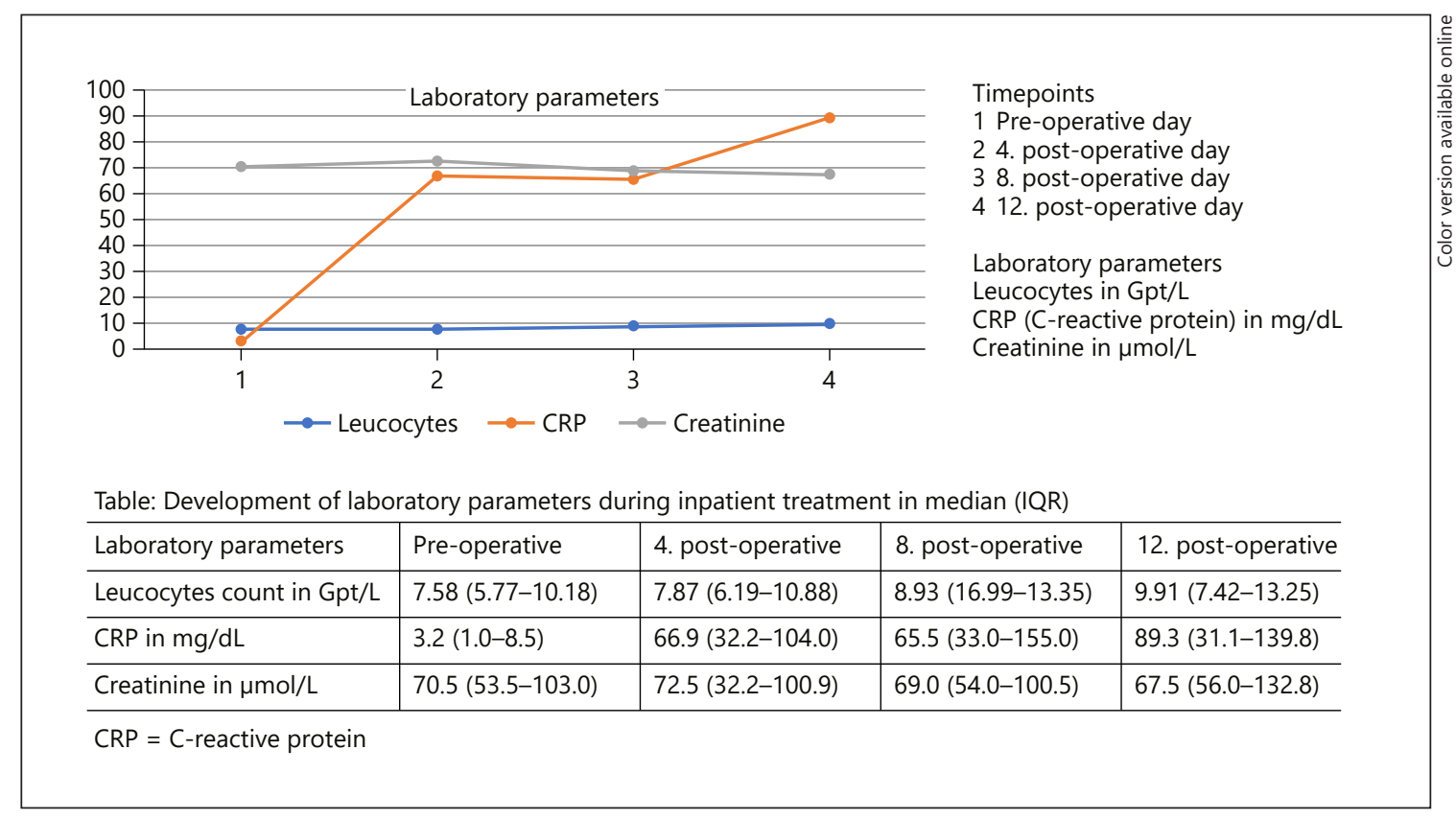

Fig. 2. Development of laboratory parameters during inpatient treatment.

significantly $\left(10^{5}\right.$ colony forming units) colonized with Staph. haemolyticus at day 12 after RC $(n=12 ; 40.0 \%)$. Figure 1 shows the development of colonization of the IC during inpatient treatment. Furthermore, we evaluated the development of the laboratory parameters leucocyte count, C-reactive protein (CRP), and creatine during inpatient treatment. This is illustrated in Figure 2. Interestingly, CRP is at the highest at day 12 following IC, while leucocyte count as well as creatinine remain in the normal range over time.

\section{Discussion}

There are sparse data on infectious complications following RC with IC, so we conducted a prospective unicentric noninterventional trial about infectious complications following RC with IC. To our knowledge, only Hara et al. [7] conducted a prospective trial with a similar topic in 2008, but the authors focused on SSI. They concluded that 1-day antimicrobial prophylaxis had an equivalent with antimicrobial prophylaxis for 3 days or more for preventing postoperative septic complications in patients undergoing RC with urinary diversion [7]. In contrast, we evaluated our in-house prophylaxis regimen prospectively for all infectious complications, only considered patients with IC as a urinary diversion in a rather short period of time ( 9 months), so we must assume that our study population is nearly homogenous. However, our study population is rather old with a median age of 70.5 years and not all patients received oncological therapy according to published guidelines prior to RC, for example, neoadjuvant chemotherapy [16], but the population might reflect the real-life situation.

Overall, $16.7 \%$ of patients had infectious complications following $\mathrm{RC}$ during the first 30 days, which is less than described in the literature [5]. Additionally, no sepsis was reported. Kolwijck et al. [6] described in their retrospective case series that the incidence of infections after $\mathrm{RC}$ is high and particularly ureteral stent removal was associated with both bacteremia and complicated UTIs. Based on the results of this study, antibiotic prophylaxis might need to be broadened for patients undergoing RC. Further research is required to investigate whether current guideline needs to be altered concerning the administration of antibiotic prophylaxis just before stent removal [6]. In our study, we observed the same, all infectious complications, especially UTIs, occurred following stent removal and we did not administer an antibiotic prophylaxis before this procedure. However, Kolwijck et al. [6] also included patients with Hautmann neobladder into their case series, and the type of urinary diversion is especially important when considering antibiotic prophylaxis. Haider et al. [9] concluded in their retrospective multicenter study that patients with continent urinary di- 
version after RC have a significantly higher risk for developing UTI. Prolonged perioperative administration of antibiotics does not seem to reduce the risk of UTI. Enterococcus, as the most commonly isolated bacteria, is not covered by the most recommended antibiotic prophylaxis regimen. Therefore, different antibiotic regimen should be considered for high-risk patients. In conclusion, further studies should evaluate antibiotic prophylaxis regimen prospectively in only 1 urinary diversion, as we did. Additionally, it is when considering the antibiotic drug essential to know your local resistance rates [6, 9], for example, we only witnessed infectious E. faecium at our institution, so it would be necessary for us to know our local resistance rates of Enterococci before deciding on an antibiotic, for example, prior to stent removal.

Who are these patients high at risk, where another prophylaxis regimen should be considered like Haider et al. [9] suggested? At least in our population, palliative cystectomy and a long time between initial diagnosis and RC are significantly associated with complicated UTI. This was not described before. Unfortunately, we were not able to identify a single risk factor for UTI in univariate Cox regression. Pariser et al. [11] described body mass index (BMI), operating room time, smoking, and total parenteral nutrition as risk factors for infection after RC. Interestingly, we were only able to report a significant linear correlation of operating time with SSI in univariate Cox regression, which is rather straightforward as an explanation for the development of SSI following surgery. Goldberg et al. [8] reported in their retrospective case series focusing on SSI that preoperative BMI reduction and maximal preoperative medical optimization to lower intensive care unit admittance rates and should be part of the ideal strategy for lowering SSI rates. This is also a straightforward explanation, and, in our opinion, optimization of the patient should be part of the medical strategy prior to every major surgery.

Interestingly, we were able to describe that the IC gets colonized with bacteria and fungi of the normal skin flora over time, which could have been expected, but was not, at least to our knowledge, described before, and we must emphasize that asymptomatic bacteria without intervention in the urinary tract does not have to be treated [18]. Consequently, the only event following RC to treat this colonization would be the stent removal, which is associated with a peak of UTI (please see above). Logically, we cannot agree with Pariser et al. [11] who suggested to also include a fungal coverage in the prophylaxis regime in RC since we did not identify any fungal infections only colonization with Candida albicans. The develop- ment of laboratory parameters in our study population is easily explained, because the peak of CRP on day 12 following RC correlates with the infectious complications, which all took place at that timepoint, so CRP might be more appropriate for the identification of infectious events at that timepoint following RC, but this needs further evaluation in prospective trials.

One last thing needs to be discussed in detail. There is a lack of adherence to published guidelines on antibiotic prophylaxis in RC, even though guideline adherence is an important approach to antibiotic stewardship and can reduce the development of multidrug-resistant pathogens $[5,13]$. What are the reasons for this? First, there is insufficient evidence for antibiotic prophylaxis in RC mainly due to the retrospective nature of most of the studies. Also, some of the available studies lack quality, for example, Wang et al. [1] excluded patients with a prolonged hospital stay or perioperative complications. Second, it is challenging to include local resistance rates into guidelines. However, most evidence for antibiotic prophylaxis in $\mathrm{RC}$ arises from the prospective study published by Hara et al. [7]; they also reported that their present study suggests that antibiotic administration on the operative day is critical for preventing infectious complications and additional postoperative use may be selectively applied, like before stent removal. This is comparable to our prospective study and should be further evaluated in randomized controlled trials.

Furthermore, even if this is a prospective study with some advantages, like the homogenous study population with IC as only urinary diversion, we must assume that our data have some limitations like a unicentric study population, that we did not include some clinical data, for example, BMI, and there was 1 violation of the study protocol since we included also patients with bladder-invasive prostate cancer. Additionally, our number of cases is still small, so multivariate analysis of those factors that showed an independent influence on both end points could not be performed.

\section{Conclusion}

In Conclusion, we have shown that bacterial colonization of IC urine from the skin flora does occur from day 12 onward. It is also likely that an evaluation of reduction of perioperative antibiotic prophylaxis is safe with perhaps additional single-shot coverage of the time of stent removal. Furthermore, rather than extensive antibiotic prophylaxis for all patients, a more calculated approach 
taking into account local resistance rates, the type of urinary diversion and the risk-profile of individual patients would be a better approach to the prevention of postoperative infectious complications after RC and IC.

\section{Statement of Ethics}

Before starting the study, we obtained the approval of the local ethics review board at the University Medical Center in Rostock (A 2019-0213 from 26 November 2019). Written informed consent was mandatory for study inclusion, so written informed consent was obtained from all participants. Furthermore, the study was registered at the German Clinical Trials Register (DKRS 00020406).

\section{Conflict of Interest Statement}

All the authors declare that they have no conflict of interest regarding this study.

\section{Funding Sources}

This project received no external funding.

\section{Author Contributions}

L.S., L.T., D.L.D., and O.W.H. participated in the design of the review and critical revision of the article. L.S., L.T., and D.L.D. participated in data acquisition, data analysis, and drafting of the article.

\section{Data Availability Statement}

All data are available upon request from the corresponding author due to ethical and legal reasons (ethics review board University Medical Center Rostock).

\section{References}

1 Wang Y, Shen W, Yuan X, Akezhouli S, Jin D, Chen $\mathrm{H}$. A perioperative management to reduce rate of urinary tract infection for patient underwent radical cystectomy with ileal conduit diversion. Int Urol Nephrol. 2021 Mar; 53(3):401-7.

2 Bochner BH, Bochner BH, Kattan MW, Vora KC. Postoperative nomogram predicting risk of recurrence after radical cystectomy for bladder cancer. J Clin Oncol. 2006 Aug 20; 24(24):3967-72.

3 Stein JP, Lieskovsky G, Cote R, Groshen S, Feng AC, Boyd S, et al. Radical cystectomy in the treatment of invasive bladder cancer: long-term results in 1054 patients. J Clin Oncol. 2001 Feb 1;19(3):666-75.

4 Colombo R, Naspro R. Ileal conduit as the standard for urinary diversion after radical cystectomy for bladder cancer. Eur Urol Suppl. 2010;25(9):736-44.

5 Krasnow RE, Mossanen M, Koo S, Kubiak DW, Preston MA, Chung BI, et al. Prophylactic antibiotics and postoperative complications of radical cystectomy: a population based analysis in the United States. J Urol. 2017 Aug;198(2):297-304.

6 Kolwijck E, Seegers AEM, Tops SCM, van der Heijden AG, Sedelaar JPM, Oever JT. Incidence and microbiology of post-operative infections after radical cystectomy and ureteral stent removal; a retrospective cohort study. BMC Infect Dis. 2019;19:303.

7 Hara N, Kitamura Y, Saito T, Kamatsubara S, Nishiyama T, Takahashi K. Perioperative antibiotics in radical cystectomy with ileal con- duit urinary diversion: efficacy and risk of antimicrobial prophylaxis on the operation day alone. BMC Infect Dis. 2019 Apr 3;19(1): 303.

8 Goldberg H, Shenhar C, Tamir H, Mano R, Baniel J, Margel D, et al. Predictors of surgical site infection after radical cystectomy: should we enhance surgical antibiotic prophylaxis? World J Urol. 2019 Jun;37(6):1137-43.

9 Haider M, Ladurner C, Mayr R, Tandogdu Z, Fritsche HM, Fradet V, et al. Use and duration of antibiotic prophylaxis and the rate of urinary tract infections after radical cystectomy for bladder cancer: results of a multicentric series. Urol Oncol. 2019 May;37(5):300.e9e15.

10 Werntz RP, Martinez-Acevedo A, Amadi H, Kopp R, La Rochelle J, Koppie T, et al. Prophylactic antibiotics following radical cystectomy reduces urinary tract infections and readmission for sepsis from a urinary source. Urol Oncol. 2018;36:238.

11 Pariser JJ, Anderson BB, Pearce SM, Han Z, Rodriguez JA 3rd, Landon E, et al. The effect of broader, directed antimicrobial prophylaxis including fungal coverage on perioperative infectious complications after radical cystectomy. Urol Oncol. 2018 May;36(5):238.e1238.e5.

12 Schneidewind L, Kranz J, Boehm K, Spachmann P, Siegel F, Huck N, et al. Antibiotic stewardship (ABS). Definition, contents, necessity and practice on examples of current clinical-urological controversies. Urologe A. 2016 Apr;55(4):489-93.
13 Cai T, Verze P, Brugnolli A, Tiscione D, Luciani LG, Eccher C, et al. Adherence to European Association of Urology Guidelines on Prophylactic Antibiotics: an important step in antimicrobial stewardship. Eur Urol. 2016 Feb;69(2):276-83.

14 Tong A, Flemming K, McInnes E, Oliver S, Craig J. Enhancing transparency in reporting the synthesis of qualitative research: ENTREQ. BMC Med Res Methodol. 2012 Nov 27;12:181.

15 The Clavien-Dindo classification. Available from: https://www.assessurgery.com/clavien-dindo-classification/. Accessed 2021 May 13.

16 The European Association of Urology (EAU) guideline on muscle-invasive and metastatic bladder cancer. Available from: https:// uroweb.org/wp-content/uploads/EAUGuidelines-on-Muscle-Invasive-and-Metastatic-Bladder-Cancer-2021.pdf. Accessed 2021 May 13.

17 KDIGO clinical practice guidelines. Available from: https://kdigo.org/guidelines/. Accessed 2021 May 13.

18 The European Association of Urology (EAU) guideline on urological infections. Available from: https://uroweb.org/wp-content/uploads/EAU-Pocket-Guidelines-on-Urological-Infections-2021.pdf. Accessed 2021 May 15.

19 Criteria of systemic inflammatory response syndrome (SIRS). Available from: https:// www.ncbi.nlm.nih.gov/books/NBK547669/. Accessed 2021 May 13. 